\title{
Doublecortin-like kinase 1 expression associates with breast cancer with neuroendocrine differentiation
}

\author{
Yu-Hong Liu' ${ }^{1}$, Julia Y.S. Tsang ${ }^{2}$, Yun-Bi Ni², Thazin Hlaing ${ }^{3}$, Siu-Ki Chan ${ }^{4}$, Kui-Fat \\ Chan $^{5}$, Chun-Wai Ko ${ }^{2}$, S. Shafaq Mujtaba ${ }^{6}$ and Gary M. Tse ${ }^{2}$ \\ ${ }^{1}$ Department of Pathology, The Affiliated Baoan Hospital of Southern Medical University, Shenzhen, China \\ 2 Department of Anatomical and Cellular Pathology, The Chinese University of Hong Kong, Hong Kong \\ ${ }^{3}$ Department of Anatomic Pathology, Centro Hospitalar Conde de Sao Januario, Macao, SAR, China \\ ${ }^{4}$ Department of Pathology, Kwong Wah Hospital, Hong Kong \\ ${ }^{5}$ Department of Pathology, Tuen Mun Hospital, Hong Kong \\ ${ }^{6}$ Histopathology Section, Laboratory Department, King Abdullah Medical City, Makkah, Kingdom of Saudi Arabia \\ Correspondence to: Gary M. Tse, email: garytse@cuhk.edu.hk
}

Keywords: doublecortin-like kinase 1, breast cancer, neuroendocrine differentiation, prognosis

Received: June 16, 2015

Accepted: November 15, 2015

Published: November 25, 2015

\section{ABSTRACT}

Doublecortin-like kinase 1 (DCLK1), a microtubule associated kinase, has recently been proposed to be a putative marker for stemness and adverse prognosis in gastrointestinal cancers. However, it is not clear whether the protein also plays similar roles in breast cancer. Here, the expression of DCLK1 was analyzed in a large cohort of invasive breast cancers (IBC) by immunohistochemistry. DCKL1 was associated with favorable clinico-pathologic features, namely lower histologic grade, absence of lymphovascular invasion, fibrotic focus, necrosis and lower pN stage ( $p \leq 0.045)$. Additionally, independent significant correlations were found with estrogen receptor and neuroendocrine markers ( $p \leq 0.019)$, implicating its relationship with IBC with neuroendocrine differentiation (IBC-NED). In the current cohort, IBC-NED showed worse outcome than luminal cancers without NED (hazard ratio=1.756, $p=0.041$ ). Interestingly, within the IBC-NED group, DCLK1 was found to be a good prognostic factor (hazard ratio $=0.288, p=0.011$ ). These findings were in contrast to those in gastrointestinal cancers, suggesting different functional roles of DCLK1 in different types of cancers. In clinical practice, NED is not routinely assessed; thus IBC-NED are not well studied. Its poor outcome and significant heterogeneity warrants more attention. DCLK1 expression could aid in the prognostication and management of this special cancer subtype.

\section{INTRODUCTION}

Doublecortin-like kinase 1 (DCLK1) is a microtubule associated kinase, containing two doublecortin domains in the $\mathrm{N}$-terminus for regulation of microtubule polymerization and a serine/threonine protein kinase domain in the $\mathrm{C}$-terminus. It also shows substantial homology to $\mathrm{Ca} 2+/$ calmodulin-dependent protein kinase. In between the $\mathrm{N}$ and $\mathrm{C}$ termini, there is a serine/prolinerich domain for mediating multiple protein-protein interactions [1]. Its function was first described in neuronal migration and development [2]. Recently, its expression has been found in colon cancers [3-5], pancreatic [6] and esophageal cancers [7].

DCLK1 has been proposed as a cancer stem cell marker for gastrointestinal cancers. DCLK1 specifically marked cancer stem cells (CSC) that self-renew and generate tumor progeny in $A p c^{\mathrm{Min} /+}$ mice [8]. DCLK1 positive differentiated tuft cells can be activated by tissue injury and initiate colon cancer [9]. In pancreatic cancers, DCLK1 positive cells displayed increased sphere forming and tumor initiating capacity [10], and enhanced epithelial- 
mesenchymal transition (EMT), a process closely linked to the acquisition of stem cell properties, via regulation of microRNA biogenesis [4, 6, 11]. Interestingly, colorectal cancer with high DCLK1 expression had increased cancer specific mortality [5]. Upregulation of DCLK1 expression in blood circulation was found in chemoradiotherapytreated colorectal cancer patients [12].

Aberrant DCLK1 expression was detected in IBC [13]. Knockdown of oncogenic miR-21 in IBC was accompanied by a decrease in DCLK1 expression [14]. Apart from these, no other information is currently available. It is not clear whether the aberrant DCLK1 expression contributed to IBC tumor aggressiveness as in gastrointestinal cancers. In addition, CSC heterogeneity exists in different breast cancer subtypes [15], and the commonly used CSC markers did not identify all CSC populations. It will be interesting to explore using DCLK1 as a CSC marker in breast cancer subtypes.

In this study, we evaluated the expression of DCLK1 in a large cohort of breast cancer by immunohistochemistry (IHC), its association with clinico-pathological features and other biomarkers (including CSC markers) expression, as well as the relationship with breast cancer outcome.

\section{RESULTS}

A total of 1132 cases were included in this cohort. The mean patients' age was $54.6 \pm 12.7$ (range 22-97) years. The mean tumor size was $2.67 \pm 1.52$ (range $0.2-13.9$ ) $\mathrm{cm}$. One hundred and seventy three cases $(15.3 \%)$ were grade I, 457 cases (40.4\%) were grade II and 502 cases $(44.3 \%)$ were grade III. Nine hundred and eighty seven cases were IBC of no special type (IBC-NST). There were 35 cases of invasive lobular cancers (ILC), 48 cases of breast cancers with medullary features, nine cases of mucinous cancers and eight cases of neuroendocrine cancers. The remaining 45 cases were of other miscellaneous histologic types, including micropapillary carcinoma, papillary carcinoma, tubular carcinomas, tubulo-lobular carcinoma and metaplastic carcinomas. Details of the clinico-pathologic features are summarized in Table 2. Overall, 418 cases (36.9\%) were DCLK1 high and 513 cases $(63.1 \%)$ were DCLK1 low. Representative staining is shown in Figure 1.

\section{Correlation with clinico-pathologic features, biomarkers and molecular subtypes}

High DCLK1 expression correlated with lower grade $(p<0.001)$, the absence of FF $(p=0.045)$, the absence of necrosis $(p=0.005)$, the absence of LVI ( $p=$ $0.004)$ and lower $\mathrm{pN}$ stage $(p=0.002)$ but not age, EIC and p T stage (Table 2).

Among the 1121 invasive cancers with complete IHC data for molecular subtypes classification, 536 (47.8\%) were luminal A, $320(28.5 \%)$ were luminal
B, 112 (10.0\%) were HER2-OE and 153 (13.7\%) were triple negative breast cancers (TNBC) (including 68 (6.1\%) BLBC and 85 (7.6\%) unclassified). The DCLK1 expression rate was $47.2 \%$ in luminal $\mathrm{A}, 34.4 \%$ in luminal B, $22.3 \%$ in HER2-OE and $17.0 \%$ in TNBC $(23.5 \%$ in BLBC and $11.8 \%$ in unclassified). DCLK1 expression showed a differential expression in different molecular subtypes with the highest prevalence in luminal cancers $(p<0.001)$ (Table 2).

In line with that, DCLK1 also correlated positively with the expression of ER and PR ( $p<0.001$ for both). Additionally, DCLK1 correlated with expression of AR ( $p$ $=0.010)$, SYN $(p<0.001)$ and CG $(<0.001)$ positively but negatively with HER2 $(p=0.001), \operatorname{Ki67}(p<0.001)$, c-Kit $(p=0.034)$, CK5/6 $(p=0.030)$ and $p$-cadherin $(p<$ $0.001)$. There was no significant correlation with EGFR, p63, CK14, CD44, ALDH1, vimentin and SOX2 (Table $3)$. By multivariate analysis, only LVI $(\mathrm{OR}=0.590, p=$ $0.001,95 \% \mathrm{CI}=0.427-0.817), \mathrm{ER}(\mathrm{OR}=2.316, p<0.001$, $95 \% \mathrm{CI}=1.648-3.255), \mathrm{CG}(\mathrm{OR}=1.611, p=0.019,95 \%$ $\mathrm{CI}=1.080-2.401)$ and $\mathrm{SYN}(\mathrm{OR}=1.655, p<0.001,95 \%$ $\mathrm{CI}=1.298-2.110$ ) were found to be independent parameters associated with DCLK1 expression (Supplementary Table S1). Similar results were obtained when DCLK1 expression was analyzed as a continuous variable, except for FF (Tables 2-3 and Supplementary Table S2).

\section{Relationship with clinico-pathologic features and biomarkers in IBC-NED}

IBC-NED was defined by $\geq 1 \%$ expression of GC and/or SYN or showing morphological NED features [16-18]. All cases with morphologic NED features in fact showed NED marker expression. According to this criteria, 135 cases $(12.1 \%)$ were classified as IBC-NED. Focal expression (1-49\%) of either neuroendocrine markers was found in 81 cases and diffuse $(\geq 50 \%)$ expression in 54 cases. Of these 135 cases, there were 112 IBC-NST. Others included 2 ILC, 4 carcinomas with medullary features, 3 mucinous carcinomas, 8 morphologic neuroendocrine carcinomas, 3 papillary carcinomas, 2 metaplastic carcinomas and one invasive micropapillary carcinoma. By molecular classification, 74 were luminal A, 54 were luminal B, 3 were HER2$\mathrm{OE}$ and 3 were unclassified. IBC-NED ( $1 \%$ cutoff) were associated with the absence of necrosis $(p=0.028)$, the presence of LVI $(p=0.005)$, older age $(p<0.001)$ and luminal cancers $(p<0.001)$. Regarding biomarkers, it also correlated positively with ER and PR $(p<0.001$ for both) and negatively with HER2 $(p=0.003), \operatorname{EGFR}(p$ $=0.033$ ), basal and EMT markers (including p63, c-kit, CK5/6, CK14, p-cadherin and vimentin; $p \leq 0.034$ ) (Supplementary Table S3). Concentrating on IBC- NED with diffuse NED marker expression (50\% cutoff), there were significant associations with necrosis, older age, 


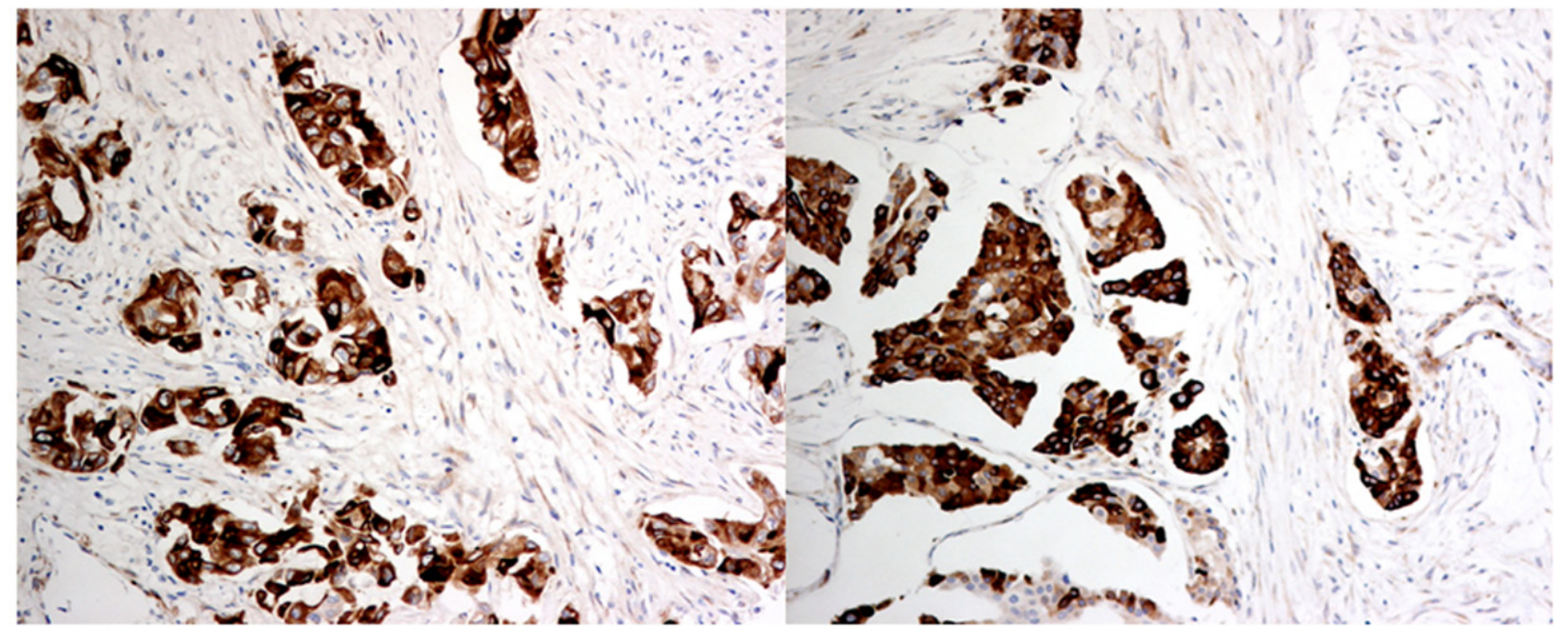

Figure 1: Representative immunohistochemical staining of DCLK1 (x400). High cytoplasmic immunoreactivity of DCLK1 in tumor cells but not in surrounding stroma. All micrographs were taken with a 40x objective, Nikon microscope equipped with a digital color camera and software.
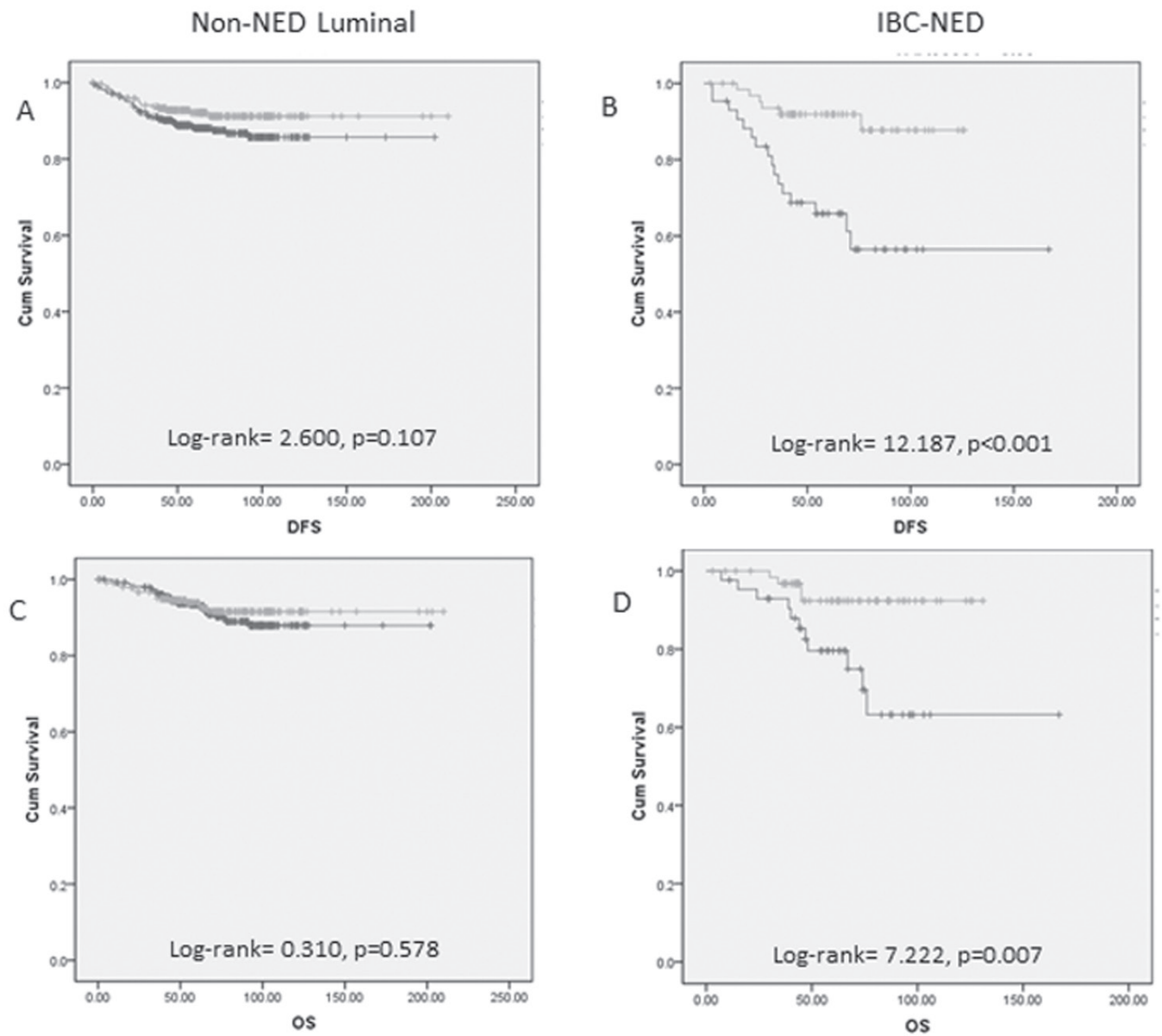

DCLK1 low

DCLK1 high

Figure 2: Kaplan-meier analysis of DFS and OS on non-NED luminal and IBC-NED cancers according to DCKL1 expression. DFS in non-NED luminal cancers A. and IBC-NED B. with different level of DCLK1 expression was compared. OS in nonNED luminal cancers $\mathbf{C}$. and IBC-NED D. with different level of DCLK1 expression was compared. DCKL1 expression was related to DFS and OS in IBC-NED but not non-NED luminal cancers. 
Table 1: Antibodies used for IHC analysis

\begin{tabular}{|c|c|c|c|c|c|c|c|}
\hline Markers & Company & Clone & Dilution & $\begin{array}{l}\text { Antigen } \\
\text { retrival }\end{array}$ & $\begin{array}{l}\text { Incubation } \\
\text { condition } \\
\left(\mathbf{m i n},{ }^{\circ} \mathrm{C}\right)\end{array}$ & Assessment & cutoff \\
\hline ER & Neomarkers & SP1 & Pre-diluted & EDTA pH8 & 32,37 & $\mathrm{~N}$ & $1 \%$ \\
\hline PR & Ventana & IE2 & Pre-diluted & EDTA pH8 & 32,37 & $\mathrm{~N}$ & $1 \%$ \\
\hline $\mathrm{AR}$ & Dako & AR441 & $1: 100$ & EDTA pH8 & 56,37 & $\mathrm{~N}$ & $1 \%$ \\
\hline Ki67 & Ventana & 41912 & Pre-diluted & EDTA pH8 & 32,37 & $\mathrm{~N}$ & $14 \%$ \\
\hline EGFR & Ventana & $3 \mathrm{C} 6$ & Pre-diluted & EDTA pH8 & 32,37 & $\mathrm{M}$ & $5 \%$ \\
\hline HER2 & Ventana & $4 \mathrm{~B} 5$ & Pre-diluted & EDTA pH8 & 16,37 & M & $3+$ \\
\hline CK5/6 & Dako & D5/16 B4 & $1: 40$ & EDTA pH8 & 32,37 & $\mathrm{C}, \mathrm{M}$ & $5 \%$ \\
\hline CK14 & Neomarkers & LL002 & $1: 100$ & EDTA pH8 & 32,37 & $\mathrm{C}, \mathrm{M}$ & $5 \%$ \\
\hline c-kit & Dako & 104D2 & $1: 300$ & EDTA pH8 & 32,37 & $\mathrm{C}, \mathrm{M}$ & $5 \%$ \\
\hline $\mathrm{P} 63$ & Ventana & $4 \mathrm{~A} 4$ & Pre-diluted & EDTA pH8 & 32,37 & $\mathrm{~N}$ & $5 \%$ \\
\hline $\begin{array}{c}\text { Synaptophysin } \\
\text { (SYN) }\end{array}$ & Novocastra & $27 \mathrm{G} 12$ & $1: 50$ & EDTA pH8 & 32,37 & $\mathrm{C}, \mathrm{M}$ & $1 \%$ \\
\hline $\begin{array}{c}\text { Chromogranin } \\
(\mathrm{CG})\end{array}$ & Biogene & $\begin{array}{c}\text { MU-126- } \\
\text { UC }\end{array}$ & $1: 200$ & EDTA pH8 & 32,37 & $\mathrm{C}, \mathrm{M}$ & $1 \%$ \\
\hline SOX2 & Ventana & SP76 & Pre-diluted & EDTA pH8 & 32,37 & $\mathrm{~N}$ & $1 \%$ \\
\hline vimentin & Dako & V9 & $1: 2000$ & EDTA pH8 & $24, \mathrm{RT}$ & $\mathrm{C}, \mathrm{M}$ & $10 \%$ \\
\hline p-cadherin & $\begin{array}{l}\mathrm{BD} \\
\text { transduction } \\
\quad \text { lab } \\
\end{array}$ & 56/p-cad & $1: 200$ & EDTA pH8 & 32,37 & $\mathrm{C}, \mathrm{M}$ & $10 \%$ \\
\hline CD44 & Ventana & SP37 & Pre-diluted & EDTA pH8 & 32,37 & $\mathrm{M}$ & $5 \%$ \\
\hline ALDH & $\begin{array}{l}\text { BD } \\
\text { transduction } \\
\quad \text { lab } \\
\end{array}$ & 44/ALDH & $1: 600$ & EDTA pH8 & 32,37 & $\mathrm{C}$ & $5 \%$ \\
\hline DCLK1 & Abcam & Polyclonal & $1: 100$ & Citrate pH6 & 32,37 & $\mathrm{C}$ & IHC score 4 \\
\hline
\end{tabular}

'N': nuclear; 'C': cytoplasmic; 'M': membraneous

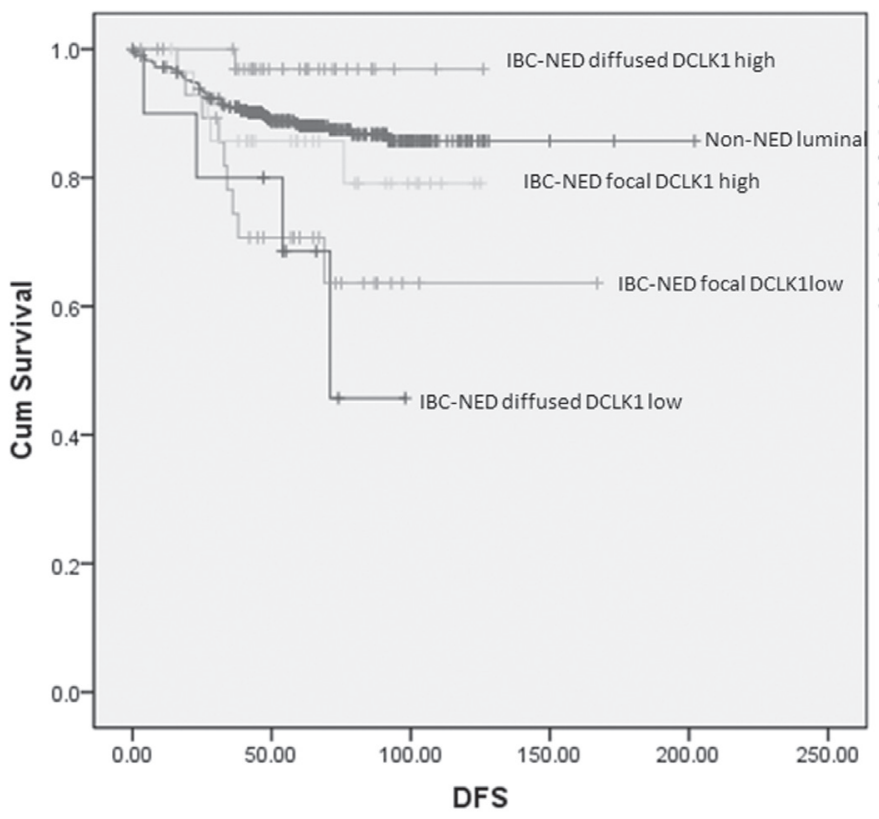

Figure 3: Kaplan-meier analysis on DFS of non-NED luminal and IBC-NED according to NED marker and DCKL1 expression. DCKL1 low IBC-NED regardless of focal or diffused NED marker expression showed worse DFS compared to non-NED luminal cancers. 
Table 2: Correlation of DCLK1 expression with clinic-pathological features

\begin{tabular}{|c|c|c|c|c|c|c|c|c|}
\hline & & DCLK1 & & & & IHC score & & \\
\hline & & lo & hi & Total & $p$-value & \begin{tabular}{|c|} 
Mean \\
(SD)
\end{tabular} & $\begin{array}{r}\text { Median } \\
\text { (IQR) }\end{array}$ & $P$-value \\
\hline \multirow[t]{4}{*}{ Grade } & 1 & 94 & 79 & 173 & $<0.001$ & $3.3(2.7)$ & $3(0-6)$ & $<0.001$ \\
\hline & 2 & 259 & 198 & 457 & & $3.0(2.6)$ & $3(0-5)$ & \\
\hline & 3 & 361 & 141 & 502 & & $2.3(2.2)$ & $2(0-4)$ & \\
\hline & Total & 714 & 418 & 1132 & & & & \\
\hline \multirow[t]{3}{*}{$\mathrm{FF}$} & Absence & 519 & 323 & 842 & 0.045 & $2.8(2.5)$ & $2(0-5)$ & 0.208 \\
\hline & Presence & 184 & 85 & 269 & & $2.6(2.4)$ & $2(0-4)$ & \\
\hline & Total & 703 & 408 & 1111 & & & & \\
\hline \multirow[t]{3}{*}{ necrosis } & Absence & 538 & 342 & 880 & 0.005 & $2.9(2.5)$ & $2(0-5)$ & $<0.001$ \\
\hline & Presence & 151 & 60 & 211 & & $2.7(2.5)$ & $2(0-4)$ & \\
\hline & Total & 689 & 402 & 1091 & & & & \\
\hline \multirow[t]{3}{*}{ EIC } & Absence & 565 & 325 & 890 & 0.680 & $2.8(2.5)$ & $2(0-5)$ & 0.741 \\
\hline & Presence & 142 & 87 & 229 & & $2.7(2.5)$ & $2(0-4)$ & \\
\hline & Total & 707 & 412 & 1119 & & & & \\
\hline \multirow[t]{3}{*}{ LVI } & Absence & 472 & 309 & 781 & 0.004 & $2.8(2.5)$ & $3(0-5)$ & 0.034 \\
\hline & Presence & 207 & 89 & 296 & & $2.5(2.2)$ & $2(0-4)$ & \\
\hline & Total & 679 & 398 & 1077 & & & & \\
\hline \multirow[t]{5}{*}{$\mathrm{pN}$} & 0 & 335 & 222 & 557 & 0.002 & $2.9(2.5)$ & $3(0-5)$ & 0.028 \\
\hline & 1 & 220 & 120 & 340 & & $2.6(2.4)$ & $2(0-4)$ & \\
\hline & 2 & 84 & 39 & 123 & & $2.5(2.3)$ & $2(0-4)$ & \\
\hline & 3 & 60 & 19 & 79 & & $2.1(2.2)$ & $2(0-3)$ & \\
\hline & Total & 699 & 400 & 1099 & & & & \\
\hline \multirow[t]{5}{*}{$\mathrm{pT}$} & 1 & 280 & 182 & 462 & 0.078 & $2.9(2.6)$ & $3(0-5)$ & 0.442 \\
\hline & 2 & 370 & 203 & 573 & & $2.6(2.4)$ & $2(0-4)$ & \\
\hline & 3 & 41 & 19 & 60 & & $2.5(2.3)$ & $2(0-4)$ & \\
\hline & 4 & 13 & 5 & 18 & & $2.0(2.0)$ & $2(0-3)$ & \\
\hline & Total & 704 & 409 & 1113 & & & & \\
\hline \multirow[t]{6}{*}{ Molecular } & Lum A & 283 & 253 & 536 & $<0.001$ & $3.3(2.6)$ & $3(0-6)$ & $<0.001$ \\
\hline & Lum B & 210 & 110 & 320 & & $2.7(2.3)$ & $2(0-4)$ & \\
\hline & HER2-OE & 87 & 25 & 112 & & $2.1(1.9)$ & $2(0-3)$ & \\
\hline & BLBC & 52 & 16 & 68 & & $2.0(2.1)$ & $2(0-4)$ & \\
\hline & $5 \mathrm{NP}$ & 75 & 10 & 85 & & $1.2(1.7)$ & $0(0-2)$ & \\
\hline & Total & 707 & 414 & 1121 & & & & \\
\hline \multirow[t]{3}{*}{ Age } & Mean & 54.1 & 55.5 & 54.6 & 0.266 & - & - & - \\
\hline & $\mathrm{SD}$ & 12.2 & 13.5 & 12.7 & & & & \\
\hline & Range & $22-94$ & $27-97$ & & & & & \\
\hline \multirow[t]{3}{*}{ Tumor size } & Mean & 2.71 & 2.60 & 2.67 & 0.076 & - & - & - \\
\hline & SD & 1.48 & 1.57 & 1.51 & & & & \\
\hline & Range & $0.3-11.0$ & $0.2-13.9$ & & & & & \\
\hline
\end{tabular}

"SD": standard deviation; "IQR": Interquartile range

ER, PR, HER2, c-kit, CK5/6 and p-cadherin, and these associations were similar to all IBC-NED (1\% cutoff) ( $p$ $\leq 0.034$ ). For LVI, EGFR and vimentin (Supplementary Table S3), the associations with all IBC-NED were not seen with IBC-NED (diffuse).

In IBC-NED, DCLK1 expression remained associated with lower grade $(p<0.001)$, lower $\mathrm{pN}$ stage $(p \leq 0.012)$, lower $\mathrm{pT}$ stage $(p \leq 0.042)$, PR positivity $(p$ $\leq 0.019)$, HER2 negativity $(p=0.002)$, low Ki67 $(p \leq$
$0.004)$ and diffuse NED expression $(p<0.001)$ regardless categorical or continuous variables analyses (Table 4).

\section{Relationship with outcome}

Follow up data were available for 987 cases with a mean follow-up duration of 65.6 months (range 1-210 months). One hundred and forty nine cases $(15.1 \%)$ had 
Table 3: Association of DCKL1 expression with biomarkers

\begin{tabular}{|c|c|c|c|c|c|c|c|c|}
\hline & & DCKL1 & & & & IHC score & & \\
\hline & & lo & hi & Total & $p$-value & Mean (SD) & $\begin{array}{l}\text { Median } \\
\text { (IQR) }\end{array}$ & $P$-value \\
\hline \multirow[t]{3}{*}{ ER } & $\mathrm{Neg}$ & 263 & 65 & 328 & $<0.001$ & $1.9(2.0)$ & $2(0-3)$ & $<0.001$ \\
\hline & Pos & 449 & 350 & 799 & & $3.1(2.5)$ & $3(0-5)$ & \\
\hline & Total & 712 & 415 & 1127 & & & & \\
\hline \multirow[t]{3}{*}{ PR } & Neg & 286 & 93 & 379 & $<0.001$ & $2.1(2.4)$ & $2(0-3)$ & $<0.001$ \\
\hline & Pos & 420 & 322 & 742 & & $3.1(2.5)$ & $3(2-5)$ & \\
\hline & Total & 706 & 415 & 1121 & & & & \\
\hline \multirow[t]{3}{*}{$\mathrm{AR}$} & Neg & 444 & 235 & 679 & 0.043 & $2.5(2.4)$ & $2(0-5)$ & 0.004 \\
\hline & Pos & 270 & 184 & 454 & & $3.0(2.4)$ & $3(0-5)$ & \\
\hline & Total & 714 & 419 & 1133 & & & & \\
\hline \multirow[t]{3}{*}{ EGFR } & Neg & 664 & 394 & 1058 & 0.297 & $2.8(2.5)$ & $2(0-5)$ & 0.793 \\
\hline & Pos & 41 & 18 & 59 & & $2.6(2.2)$ & $2(0-5)$ & \\
\hline & Total & 705 & 412 & 1117 & & & & \\
\hline \multirow[t]{3}{*}{ HER2 } & Neg & 552 & 358 & 910 & 0.001 & $2.9(2.5)$ & $3(0-5)$ & 0.002 \\
\hline & Pos & 158 & 59 & 217 & & $2.2(2.0)$ & $2(0-4)$ & \\
\hline & Total & 710 & 417 & 1127 & & & & \\
\hline \multirow[t]{3}{*}{ Ki67 } & low & 402 & 289 & 691 & $<0.001$ & $3.0(2.5)$ & $3(0-6)$ & $<0.001$ \\
\hline & high & 302 & 126 & 428 & & $2.4(2.3)$ & $2(0-4)$ & \\
\hline & Total & 704 & 415 & 1119 & & & & \\
\hline \multirow[t]{3}{*}{ c-KIT } & Neg & 578 & 359 & 937 & 0.034 & $2.8(2.5)$ & $2(0-5)$ & 0.157 \\
\hline & Pos & 124 & 53 & 177 & & $2.5(2.2)$ & $2(0-4)$ & \\
\hline & Total & 702 & 412 & 1114 & & & & \\
\hline \multirow[t]{3}{*}{ P63 } & $\mathrm{Neg}$ & 674 & 399 & 1073 & 0.470 & $2.8(2.5)$ & $2(0-5)$ & 0.888 \\
\hline & Pos & 30 & 14 & 44 & & $2.6(2.1)$ & $2(2-5)$ & \\
\hline & Total & 704 & 413 & 1117 & & & & \\
\hline \multirow[t]{3}{*}{ CK5/6 } & Neg & 617 & 381 & 998 & 0.030 & $2.8(2.5)$ & $3(0-5)$ & 0.022 \\
\hline & Pos & 85 & 33 & 118 & & $2.2(2.1)$ & $2(0-4)$ & \\
\hline & Total & 702 & 414 & 1116 & & & & \\
\hline \multirow[t]{3}{*}{ CK14 } & Neg & 658 & 390 & 1048 & 0.465 & $2.8(2.5)$ & $2(0-5)$ & 0.313 \\
\hline & Pos & 47 & 23 & 70 & & $2.4(2.1)$ & $2(0-4)$ & \\
\hline & Total & 705 & 413 & 1118 & & & & \\
\hline \multirow[t]{3}{*}{ SYN } & Neg & 659 & 329 & 988 & $<0.001$ & $2.5(2.4)$ & $2(0-4)$ & $<0.001$ \\
\hline & Pos & 44 & 85 & 129 & & $4.5(2.4)$ & $5(4-7)$ & \\
\hline & Total & 703 & 414 & 1117 & & & & \\
\hline \multirow[t]{3}{*}{$\mathrm{CG}$} & $\mathrm{Neg}$ & 690 & 377 & 1067 & $<0.001$ & $2.7(2.4)$ & $2(0-5)$ & $<0.001$ \\
\hline & Pos & 11 & 39 & 50 & & $5.2(2.3)$ & $6(4-7)$ & \\
\hline & Total & 701 & 416 & 1117 & & & & \\
\hline \multirow[t]{3}{*}{$\mathrm{SYN} /$ and $\mathrm{CG}$} & Neg & 650 & 325 & 975 & $<0.001$ & $2.5(2.4)$ & $2(0-4)$ & $<0.001$ \\
\hline & Pos & 47 & 88 & 135 & & $4.5(2.4)$ & $5(3-7)$ & \\
\hline & Total & 697 & 413 & 1110 & & & & \\
\hline \multirow[t]{3}{*}{ p-Cadherin } & $\mathrm{Neg}$ & 503 & 343 & 846 & $<0.001$ & $3.0(2.5)$ & $3(0-5)$ & $<0.001$ \\
\hline & Pos & 192 & 64 & 256 & & $2.1(2.1)$ & $2(0-4)$ & \\
\hline & Total & 695 & 407 & 1102 & & & & \\
\hline \multirow[t]{3}{*}{ Vimentin } & $\mathrm{Neg}$ & 604 & 354 & 958 & 0.928 & $2.8(2.5)$ & $3(0-5)$ & 0.670 \\
\hline & Pos & 94 & 56 & 150 & & $2.7(2.4)$ & $2.5(0-4)$ & \\
\hline & Total & 698 & 410 & 1108 & & & & \\
\hline \multirow[t]{3}{*}{ CD44 } & Neg & 217 & 135 & 352 & 0.895 & $2.8(2.5)$ & $3(0-5)$ & 0.180 \\
\hline & Pos & 105 & 67 & 172 & & $3.1(2.4)$ & $3(1-5)$ & \\
\hline & Total & 322 & 202 & 524 & & & & \\
\hline
\end{tabular}




\begin{tabular}{|l|l|l|l|l|l|l|l|l|}
\hline ALDH & Neg & 305 & 187 & 492 & 0.569 & $2.9(2.5)$ & $3(0-5)$ & 0.725 \\
\hline & Pos & 20 & 15 & 35 & & $3.1(2.6)$ & $2(2-5)$ & \\
\hline & Total & 325 & 202 & 527 & & & & \\
\hline SOX2 & Neg & 260 & 162 & 422 & 0.901 & $2.9(2.5)$ & $3(0-5)$ & 0.903 \\
\hline & Pos & 64 & 41 & 105 & & $2.9(2.3)$ & $3(0-4.75)$ & \\
\hline & Total & 324 & 203 & 527 & & & & \\
\hline
\end{tabular}

"SD": standard deviation; "IQR": Interquartile range

breast cancer mortality or relapse. Among them, 116 cases $(11.8 \%)$ had breast cancer specific mortality. DCLK1 expression was associated with significantly better OS $(\log$-rank $=5.753, p=0.016)$ and DFS $(\log$-rank $=12.104$, $p=0.001)$. When segregating the cases into luminal and non-luminal cancers, DCLK1 expression was associated with better DFS significantly in luminal cancers (logrank $=5.883, p=0.015$ ) but not in non-luminal cancers (log-rank $=0.389, p=0.533$ ).

It appears that the association of DCLK1 with outcome in luminal cancers is mainly related to the relationship with IBC-NED, which are clustered within the luminal group of cancers. Analysis of the prognostic impact of DCLK1 in the IBC-NED cases and nonNED luminal cases found that DCLK1 expression was associated with better DFS (log-rank $=12.187, p$ $<0.001)$ and OS (log-rank=7.222, $p=0.007)$ in IBCNED but not in non-NED luminal cancers (Figure 2). Recent investigations suggested adverse prognosis of IBC-NED [16, 19]. This study also showed worse OS $(\log$-rank $=4.658, p=0.031)$ and DFS $(\log -\mathrm{rank}=9.294$, $p=0.002)$ in IBC-NED than luminal cancers without NED. The prognostic impact on DFS of IBC-NED was independent of ER, PR, HER2, Ki67, grade, age, tumor size and nodal involvement $(\mathrm{HR}=1.756, p=0.041$ with reference to non-NED luminal). Interestingly, those IBD-NED with worse outcome showed focal but not diffuse NED expression (supplementary figure 1S). As DCLK1 was associated with diffuse NED expression, we then compared the patients' parameters and outcome based on groupings stratified by different levels of neuroendocrine and DCLK1 expression. IBC-NED with low DCLK1 expression showed the lowest DFS rate, when compared to non-NED luminal cancers regardless of the neuroendocrine expression pattern (DCLK1 low/ neuroendocrine focal: log-rank $=8.861, p=0.003$; DCLK1 low/ neuroendocrine diffuse: $\log$-rank $=7.211, p$ $=0.007)$ (Figure 3$)$. Of note, DCLK1 was found also to have independent favorable prognostic impact on DFS of IBC-NED $(\mathrm{HR}=0.288, p=0.011,95 \% \mathrm{CI}=0.111-0.748)$ after adjustment of grade, age, tumor size, LVI, pN stage, HER2, Ki67, PR and neuroendocrine markers expression (Supplementary Table S4).

\section{DISCUSSION}

In this study, the expression of DCLK1 in a large cohort of breast cancer was analyzed. In contrast to its cancer initiating roles in gastrointestinal tumors, DCLK1 expression in breast cancer did not appear to be related to stem cell features and aggressive behavior. No positive correlation was observed with other breast CSC markers. Nonetheless, DCLK1 was negatively correlated with grade, basal (c-kit and CK5/6) as well as EMT (p-cadherin) markers. DCLK1 was more frequently found in luminal cancers than basal-like and HER2-OE subtypes $[15,20,21]$, associated with IBC-NED and potentially an independent favorable prognostic factor in these cancers.

The diagnosis of IBC-NED has been controversial. It was first defined by the presence of morphologic features similar to those of neuroendocrine tumors of the gastrointestinal tract or of the lung. However, classic neuroendocrine morphology is comparatively rare in breast cancer, thus its significance has been debatable for some time. Recently, a formal categorization of this tumor in WHO classification has been established. Apart from the morphologic features (including well-differentiated neuroendocrine tumors and poorly differentiated neuroendocrine carcinoma or small cell carcinoma), it has been defined with the expression of NED markers in over $50 \%$ of the tumor cell population in the $2003 \mathrm{WHO}$ classification [22]. In the latest 2012 classification [18], it was revised to include all tumors expressing NED markers to a greater or lesser degree. The prognostic significance of NED per se in IBC-NED has also been uncertain, with reports suggested no effects on prognosis [23-25], better [26, 27] or worse [16, 19] prognosis. In the current study, the latest WHO criteria were adopted for diagnosis of IBC-NED [18]. In agreement with the others $[16,19,28,29]$, IBC-NED was mainly associated with luminal cancers. However, IBC-NED was not associated with low tumor grade or favorable outcome as would be expected for luminal cancers. This result corroborated with heterogeneity in IBC-NED, which encompass both low grade special subtypes and aggressive high grade cancers [17]. In contrast to the previous reports showing the poor prognosis of diffuse neuroendocrine markers expression [19], this study showed poorer outcome in patients with focal rather than diffuse NED expression in IBC-NED. One study applied similar criteria demonstrated 
Table 4: Association of DCLK1 with clinico-pathological features and biomarkers in IBC-NED

\begin{tabular}{|c|c|c|c|c|c|c|c|c|}
\hline & & \begin{tabular}{|l|} 
DCLK1 \\
\end{tabular} & & & & IHC score & & \\
\hline & & lo & hi & Total & $p$-value & Mean & Median & $p$-value \\
\hline \multirow[t]{4}{*}{ Grade } & 1 & 2 & 14 & 16 & $<0.001$ & $5.8(1.8)$ & $7(4.5-7)$ & $<0.001$ \\
\hline & 2 & 17 & 52 & 69 & & $4.9(2.2)$ & $6(3.5-7)$ & \\
\hline & 3 & 28 & 22 & 50 & & $3.3(2.4)$ & $3(1.5-6)$ & \\
\hline & Total & 47 & 88 & 135 & & & & \\
\hline \multirow[t]{3}{*}{$\mathrm{FF}$} & Absence & 34 & 75 & 109 & 0.079 & $4.6(2.4)$ & $5(2.5-7)$ & 0.366 \\
\hline & Presence & 12 & 12 & 24 & & $4.1(2.4)$ & $3.5(2.5-6.5)$ & \\
\hline & Total & 46 & 87 & 133 & & & & \\
\hline \multirow[t]{3}{*}{ necrosis } & Absence & 39 & 76 & 115 & 0.440 & $4.5(2.4)$ & $5(3-7)$ & 0.463 \\
\hline & Presence & 7 & 9 & 16 & & $3.9(2.9)$ & $4(0.5-7)$ & \\
\hline & Total & 46 & 85 & 131 & & & & \\
\hline \multirow[t]{3}{*}{ EIC } & Absence & 39 & 71 & 110 & 0.645 & $4.4(2.5)$ & 5 & 0.841 \\
\hline & Presence & 7 & 16 & 23 & & $4.7(2.1)$ & 5 & \\
\hline & Total & 46 & 87 & 133 & & & & \\
\hline \multirow[t]{3}{*}{ LVI } & Absence & 20 & 59 & 79 & 0.006 & $4.8(2.3)$ & $5(3-7)$ & 0.093 \\
\hline & Presence & 24 & 25 & 49 & & $4.0(2.5)$ & $4(2-7)$ & \\
\hline & Total & 44 & 84 & 128 & & & & \\
\hline \multirow[t]{5}{*}{$\mathrm{pN}$} & 0 & 16 & 47 & 63 & 0.001 & $4.9(2.4)$ & $6(3-7)$ & 0.012 \\
\hline & 1 & 15 & 25 & 40 & & $4.2(2.3)$ & $4(2-6)$ & \\
\hline & 2 & 6 & 7 & 13 & & $4.0(2.5)$ & $4(2-6.5)$ & \\
\hline & 3 & 10 & 4 & 14 & & $2.9(2.1)$ & $3(1.5-4)$ & \\
\hline & Total & 47 & 83 & 130 & & & & \\
\hline \multirow[t]{5}{*}{$\mathrm{pT}$} & 1 & 14 & 39 & 53 & 0.028 & $5.0(2.3)$ & $6(3-7)$ & 0.042 \\
\hline & 2 & 29 & 42 & 71 & & $4.0(2.5)$ & $4(2-6)$ & \\
\hline & 3 & 1 & 2 & 3 & & $5.3(2.1)$ & $6(4.5-6.5)$ & \\
\hline & 4 & 3 & 1 & 4 & & $2.8(2.5)$ & $2.5(1-4.5)$ & \\
\hline & Total & 47 & 84 & 131 & & & & \\
\hline \multirow[t]{6}{*}{ Molecular } & Lum A & 14 & 60 & 74 & $<0.001$ & $5.2(2.1)$ & $6(4-7)$ & $<0.001$ \\
\hline & Lum B & 28 & 26 & 54 & & $3.5(2.4)$ & $3(2-6)$ & \\
\hline & HER2-OE & 3 & 0 & 3 & & $1.7(1.5)$ & $2(0-3)$ & \\
\hline & BLBC & 0 & 0 & 0 & & - & - & \\
\hline & $5 \mathrm{NP}$ & 2 & 1 & 3 & & $3.0(3.0)$ & $3(0-3)$ & \\
\hline & Total & 47 & 87 & 134 & & & 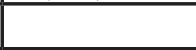 & \\
\hline \multirow[t]{3}{*}{ Age } & Mean & 56.6 & 59.7 & 58.7 & 0.249 & - & - & - \\
\hline & SD & 13.0 & 14.0 & 13.7 & & - & - & \\
\hline & Range & $30-80$ & $31-83$ & & & & & \\
\hline \multirow[t]{3}{*}{ Tumor size } & Mean & 2.86 & 2.58 & 2.68 & 0.208 & - & - & - \\
\hline & SD & 1.35 & 1.27 & 1.31 & & - & - & \\
\hline & Range & $1.0-7.0$ & $0.8-8.0$ & & & & & \\
\hline \multicolumn{9}{|l|}{ Biomarkers } \\
\hline \multirow[t]{3}{*}{ ER } & $\mathrm{Neg}$ & 6 & 3 & 9 & 0.065 & $2.8(2.5)$ & $3(0-5.5)$ & 0.031 \\
\hline & Pos & 41 & 85 & 126 & & $4.6(2.3)$ & $5(3-7)$ & \\
\hline & Total & 47 & 88 & 135 & & & & \\
\hline \multirow[t]{3}{*}{ PR } & Neg & 14 & 11 & 25 & 0.020 & $3.6(2.2)$ & $3(2-5.5)$ & 0.019 \\
\hline & Pos & 33 & 77 & 110 & & $4.7(2.4)$ & $5(3-7)$ & \\
\hline & Total & 47 & 88 & 135 & & & & \\
\hline \multirow[t]{3}{*}{$\mathrm{AR}$} & Neg & 28 & 52 & 80 & 0.957 & $5.2(2.1)$ & $5(3-7)$ & 0.458 \\
\hline & Pos & 19 & 36 & 55 & & $4.7(2.4)$ & $5(2-7)$ & \\
\hline & Total & 47 & 88 & 135 & & & & \\
\hline EGFR & Neg & 47 & 86 & 133 & 0.543 & $4.4(2.4)$ & $5(3-7)$ & 0.534 \\
\hline
\end{tabular}




\begin{tabular}{|c|c|c|c|c|c|c|c|c|}
\hline & Pos & 0 & 2 & 2 & & $5.5(2.2)$ & $5.5(4-7)$ & \\
\hline & Total & 47 & 88 & 135 & & & & \\
\hline \multirow[t]{3}{*}{ HER2 } & Neg & 37 & 84 & 121 & 0.002 & $4.6(2.3)$ & $6(3-7)$ & 0.002 \\
\hline & Pos & 10 & 3 & 13 & & $3.7(2.4)$ & $3(0-4)$ & \\
\hline & Total & 47 & 87 & 134 & & & & \\
\hline \multirow[t]{3}{*}{ Ki67 } & low & 21 & 63 & 84 & 0.002 & $4.9(2.3)$ & $6(3.5-7)$ & 0.004 \\
\hline & high & 26 & 25 & 51 & & $3.7(2.4)$ & $3(2-6)$ & \\
\hline & Total & 47 & 88 & 135 & & & & \\
\hline \multirow[t]{3}{*}{$\mathrm{c}-\mathrm{KIT}$} & Neg & 41 & 83 & 124 & 0.152 & $4.5(2.5)$ & $5(2-7)$ & 0.501 \\
\hline & Pos & 6 & 5 & 11 & & $4.2(1.8)$ & $3(3-6)$ & \\
\hline & Total & 47 & 88 & 135 & & & & \\
\hline \multirow[t]{3}{*}{ P63 } & Neg & 45 & 88 & 133 & 0.343 & $4.5(2.4)$ & $5(3-7)$ & 0.263 \\
\hline & Pos & 1 & 0 & 1 & & 2 & 2 & \\
\hline & Total & 46 & 88 & 134 & & & & \\
\hline \multirow[t]{3}{*}{ CK5/6 } & $\mathrm{Neg}$ & 46 & 86 & 132 & 0.546 & $4.4(2.4)$ & $5(3-7)$ & 0.238 \\
\hline & Pos & 0 & 2 & 2 & & $6.5(0.7)$ & $6.5(6-7)$ & \\
\hline & Total & 48 & 88 & 134 & & & & \\
\hline \multirow[t]{3}{*}{ CK14 } & Neg & 46 & 87 & 133 & 1.0 & $4.5(2.4)$ & $5(3-7)$ & 0.933 \\
\hline & Pos & 1 & 1 & 2 & & $4.5(3.5)$ & $4.5(2-4.5)$ & \\
\hline & Total & 47 & 88 & 135 & & & & \\
\hline \multirow[t]{3}{*}{ CG/SYN } & $<50 \%$ & 37 & 40 & 77 & $<0.001$ & $3.7(2.4)$ & $4(2-7)$ & $<0.001$ \\
\hline & $\geq 50 \%$ & 10 & 48 & 58 & & $5.5(2.1)$ & $7(5-7)$ & \\
\hline & Total & 47 & 88 & 135 & & & & \\
\hline \multirow[t]{3}{*}{ p-Cadherin } & $\mathrm{Neg}$ & 39 & 79 & 118 & 0.182 & $4.5(2.4)$ & $5(3-7)$ & 0.158 \\
\hline & Pos & 8 & 8 & 16 & & $3.8(2.4)$ & $3.5(2-6)$ & \\
\hline & Total & 47 & 87 & 134 & & & & \\
\hline \multirow[t]{3}{*}{ Vimentin } & Neg & 46 & 81 & 127 & 0.421 & $4.4(2.4)$ & $5(2-7)$ & 0.359 \\
\hline & Pos & 1 & 6 & 7 & & $5.4(1.5)$ & $6(4-7)$ & \\
\hline & Total & 47 & 87 & 134 & & & & \\
\hline \multirow[t]{3}{*}{ CD44 } & $\mathrm{Neg}$ & 8 & 24 & 32 & 1.0 & $5.3(2.2)$ & $6(3.5-7)$ & 0.376 \\
\hline & Pos & 5 & 13 & 21 & & $4.7(2.3)$ & $5(3.5-7)$ & \\
\hline & Total & 13 & 40 & 53 & & & & \\
\hline \multirow[t]{3}{*}{ ALDH1 } & Neg & 12 & 38 & 50 & 1.0 & $5.0(2.2)$ & $64-7)$ & 0.573 \\
\hline & Pos & 1 & 2 & 3 & & $5.7(2.3)$ & $7(3-7)$ & \\
\hline & Total & 13 & 40 & 53 & & & & \\
\hline \multirow[t]{3}{*}{ SOX2 } & Neg & 8 & 32 & 40 & 0.179 & $5.2(2.2)$ & $6(4-7)$ & 0.261 \\
\hline & Pos & 5 & 8 & 13 & & $4.5(2.3)$ & $4(3-7)$ & \\
\hline & Total & 13 & 40 & 53 & & & & \\
\hline
\end{tabular}

"SD": standard deviation; "IQR": Interquartile range

similar poor outcomes in IBC with diffuse and focal neuroendocrine marker expression [16] compared to nonNED IBC cases. Of note, when the non-NED IBC were further classified into luminal and non-luminal cases, IBCNED in fact showed an intermediate outcome, which was better than non-luminal but worse than non-NED luminal cancers. Possibly the heterogeneity in the cohort and variation in diagnostic criteria contributed to the variation in its prognostication.

As IBC-NED is heterogeneous, factors that further stratify IBC-NED into different prognostic groups could be useful for the management of these cancers. DCLK1 expression was found to be an independent favorable prognostic factor for DFS in IBC-NED regardless of the NED expression pattern. Currently, there are no specific recommendations for treatment of IBC-NED and these cases are treated as IBC-NOS. As IBC-NED are mostly luminal, they are treated with either endocrine therapy alone or together with adjuvant chemotherapy, with the latter depending on risk assessment. The additional independent prognostic impact of DCLK1 could be crucial for therapeutic decision to identify IBC-NED with favorable outcome, and sparing patients from chemotherapy. The mechanism of its good prognostic 
impact of DCLK1 in IBC-NED has not yet been explored. A recent report suggested that DCLK1 can antagonize Runx2 [30]. Runx2 has been shown to be a regulator of epithelial cell fate in mammary gland development and breast cancer [31]. Overexpression of Runx2 drove EMT-like changes in normal mammary epithelial cells, whereas its deletion in basal breast cancer cells inhibited cellular phenotypes associated with tumorigenesis [32]. In fact, in our preliminary study on Runx 2 expression, we observed a significantly higher expression of basal marker in DCLK1loRunx2hi cases compared to the others (data not shown). Interestingly, there are also interactions between them in IBC-NED patients' outcome. Cases with DCLK1loRunx2hi expression were found to have significantly worse DFS than other subgroups (data not shown). Our data may suggest that DCLK1 could at least partly act via Runx2.

In summary, DCLK1 was found to be a good prognostic factor in breast cancer, particularly in IBCNED. The result was in contrast to its tumor promoting roles in gastrointestinal cancers, suggesting different functional roles of DCLK1 in different type of cancers. In addition, using the current WHO classification, we found that IBC-NED showed a worse outcome, attributable in part to tumor heterogeneity. DCLK1 expression was shown to stratify IBC-NED into different prognostic groups. The findings could aid in the prognostication and management of this special type of IBC.

\section{MATERIALS AND METHODS}

\section{Patients data}

The histologic files of the three involved institutions were searched for IBC over periods of 2 (03-04), 4 (2002$05)$ and 7 (2003-09) years. All consecutive cases with excision specimens were included. All the specimens were formalin fixed, paraffin embedded and routinely processed. The 4 micron slides were stained with $H \& E$ and reviewed by two of the authors. The tumors were graded using modified Bloom and Richardson grading [16] and the histologic diagnosis was confirmed (WHO [17]). Invasive breast cancers with neuroendocrine differentiation (IBCNED) were defined by the presence of neuroendocrine morphological features (neuroendocrine carcinoma) and the presence of neuroendocrine differentiation (NED) (neuroendocrine marker positivity) [17]. IBC was considered as having NED if $\geq 1 \%$ tumor cells showed any expression of GC and/or SYN or showing morphologic features of NED [18, 19]. In addition, lymphovascular invasion (LVI), the presence of extensive in situ components (EIC) and fibrotic focus (FF) were also evaluated as present or absent as previously reported [20]. Patient details and clinical information were retrieved from the medical records including patients' age, tumor size, $\mathrm{pN}$ stage, $\mathrm{pT}$ stage and patient outcome data. For the outcome data, overall survival (OS) was defined as the time interval from the date of initial diagnosis to the date of breast cancer related death. Disease free survival (DFS) was defined as the duration from the date of initial diagnosis to the first detection of breast cancer specific relapse or death. The study was approved by Joint Chinese University of Hong Kong- New Territories East Cluster clinical research ethics committee.

\section{Tissue microarray construction}

Cellular areas of the tumors on H\&E slides were chosen and the corresponding areas were taken from the paraffin blocks for tissue microarray (TMA) construction. Two $0.6 \mathrm{~mm}$ tissue cores were obtained from each case. One additional core was taken from available nodal metastases. The TMAs were assembled with a tissue arrayer (Beecher Instruments, Silver Springs, MD). Sixty two composite TMA blocks, each containing maximum 54 tissue cores, were constructed. Serial 4 micron sections were cut and transferred to Superfrost Plus glass slides (Menzel-Glaser, Germany). One section from each tissue array block was stained with H\&E to confirm the presence of representative tumors in the TMA blocks.

\section{Immunohistochemistry and scoring}

The TMA slides were assessed for the different groups of biomarkers, in addition to DCLK1. The first group were steroid hormone receptors (estrogen receptor (ER), progesterone receptor (PR), and androgen receptor (AR)). The second group were growth factor receptors (human epidermal growth factor receptor 2 (HER2), epidermal growth factor receptor (EGFR)) and a proliferation marker (Ki67). The other groups were neuroendocrine markers (chromogranin (CG) and synpatophysin (SYN)), basal markers (p63, c-kit, CK14 and CK5/6), cancer stem cell markers (CD44, SOX2 and ALDH1) and EMT markers (vimentin and p-cadherin). IHC of all markers was performed by BenchMark XT automated slide-staining instrument (Ventana, Arizona, USA) with Ultraview Universal DAB Detection Kit (Ventana, Arizona, USA) after deparaffinization, rehydration and antigen retrieval. After primary antibody incubation, the sections were incubated with anti-mouse horseradish peroxidase labeled polymer (Roche, Arizona, USA) for $30 \mathrm{~min}$ at room temperature, and then developed with diaminobenzidine. All slides were counterstained with hematoxylin. The TMA slides were scored for the intensity of staining in the nucleus, cytoplasm or membrane according to different antibodies by two of the authors blinded to the clinical information and the staining results of other markers. Details of the antibodies, antigen 
retrieval, staining conditions and scoring were listed in Table 1. For DCLK1 staining, the reactivity assessed was cytoplasmic. DCLK1 was assessed for both intensity and proportion of positively stained cells. The staining intensity was graded from 0 to 3 whereas the proportion of stained cells was scored on a scale of $0-4(0=$ no detectable staining, $1=1-25 \%$ positive cells, $2=26-50 \%$ positive cells, $3=51-75 \%$ positive cells and $4=$ over $75 \%$ positive cells). An immunoscore was obtained by adding the intensity score and the percentage score. Positivity for DCLK1 was defined using the mean immunoscore as the cutoff. Immunoscore of 0-3 was regarded as negative and $>3$ as positive. Any discrepancies were resolved by reviewing at a multi-head microscope to reach a consensus.

The tumors were also classified into the 5 different molecular subtypes by immunohistochemical expression as surrogate as follows [21]:

Luminal A: ER+ and/or PR+, HER2-, CK5/6 +/- and Ki67 < 14\%

Luminal B: ER+ and/or PR+, CK5/6+/-, HER2+ or $\mathrm{Ki} 67 \geq 14 \%$

HER2 over-expressed (HER2-OE): ER-, PR-, HER2+, CK5/6 +/-

Basal like breast cancers (BLBC): ER-, PR-, HER2, (triple negative), CK5/6+ and/or EGFR+

Unclassified: ER-, PR-, HER2-, (triple negative), CK5/6- and EGFR-

\section{Statistical analysis}

The findings were analyzed using the statistical software SPSS for Windows, Version 18. Chi-square analysis or Fisher's exact test were used to test for the association of DCLK1 expression with tumor grade, FF, LVI, EIC, pN, pT, molecular subtypes and biomarker expression. Mann-Whitney U test was used for analyzing the differences in patient's age and tumor size with DCLK1 expression. The relationship of DCLK1 as a continuous variable with various clinico-pathologic features and biomarker expression was also analyzed with Mann-Whitney U test (for categorical data) and spearman correlation (rs) (for continuous variables). Survival data were evaluated with Kaplan Meier analysis and Cox regression analysis using the backward Wald method. Statistical significance was established at $p<0.05$.

\section{FUNDING}

YL was supported by NSFC (Grants no. 81001110 and 81472316).

\section{Authors' contributions}

YL performed the experiments, analyzed the data and wrote the manuscript; JT analyzed the data and wrote the manuscript; YN, SC, KC, collected and arranged the clinical data; $\mathrm{CK}$, TH performed the experiments; GT conceived the idea of the project, provided guidance and critically revised the paper. All authors read and approved the final version to be published.

\section{CONFLICTS OF INTEREST}

The authors declare no conflict of interest.

\section{REFERENCES}

1. Ohmae S, Takemoto-Kimura S, Okamura M, AdachiMorishima A, Nonaka M, Fuse T, Kida S, Tanji M, Furuyashiki T, Arakawa Y, Narumiya S, Okuno H and Bito H. Molecular identification and characterization of a family of kinases with homology to Ca2+/calmodulin-dependent protein kinases I/IV. J Biol Chem. 2006; 281:20427-20439.

2. Weimer JM and Anton ES. Doubling up on microtubule stabilizers: synergistic functions of doublecortin-like kinase and doublecortin in the developing cerebral cortex. Neuron. 2006; 49:3-4.

3. May R, Riehl TE, Hunt C, Sureban SM, Anant S and Houchen $\mathrm{CW}$. Identification of a novel putative gastrointestinal stem cell and adenoma stem cell marker, doublecortin and CaM kinase-like-1, following radiation injury and in adenomatous polyposis coli/multiple intestinal neoplasia mice. Stem Cells. 2008; 26:630-637.

4. Sureban SM, May R, Ramalingam S, Subramaniam D, Natarajan G, Anant S and Houchen CW. Selective blockade of DCAMKL-1 results in tumor growth arrest by a Let-7a MicroRNA-dependent mechanism. Gastroenterology. 2009; 137:649-659, 659 e641-642.

5. Gagliardi G, Goswami M, Passera R and Bellows CF. DCLK1 immunoreactivity in colorectal neoplasia. Clin Exp Gastroenterol. 2012; 5:35-42.

6. Sureban SM, May R, Lightfoot SA, Hoskins AB, Lerner M, Brackett DJ, Postier RG, Ramanujam R, Mohammed A, Rao CV, Wyche JH, Anant S and Houchen CW. DCAMKL-1 regulates epithelial-mesenchymal transition in human pancreatic cells through a miR-200a-dependent mechanism. Cancer Res. 2011; 71:2328-2338.

7. Vega KJ, May R, Sureban SM, Lightfoot SA, Qu D, Reed A, Weygant N, Ramanujam R, Souza R, Madhoun M, Whorton J, Anant S, Meltzer SJ and Houchen CW. Identification of the putative intestinal stem cell marker doublecortin and CaM kinase-like-1 in Barrett's esophagus and esophageal adenocarcinoma. J Gastroenterol Hepatol. 2012; 27:773-780.

8. Nakanishi Y, Seno H, Fukuoka A, Ueo T, Yamaga Y, 
Maruno T, Nakanishi N, Kanda K, Komekado H, Kawada M, Isomura A, Kawada K, Sakai Y, Yanagita M, Kageyama R, Kawaguchi Y, et al. Dclk1 distinguishes between tumor and normal stem cells in the intestine. Nat Genet. 2013; 45:98-103.

9. Westphalen CB, Asfaha S, Hayakawa Y, Takemoto Y, Lukin DJ, Nuber AH, Brandtner A, Setlik W, Remotti H, Muley A, Chen X, May R, Houchen CW, Fox JG, Gershon $\mathrm{MD}$, Quante $\mathrm{M}$, et al. Long-lived intestinal tuft cells serve as colon cancer-initiating cells. J Clin Invest. 2014; 124:1283-1295.

10. Bailey JM, Alsina J, Rasheed ZA, McAllister FM, Fu YY, Plentz R, Zhang H, Pasricha PJ, Bardeesy N, Matsui W, Maitra A and Leach SD. DCLK1 marks a morphologically distinct subpopulation of cells with stem cell properties in preinvasive pancreatic cancer. Gastroenterology. 2014; 146:245-256.

11. Chandrakesan P, Weygant N, May R, Qu D, Chinthalapally HR, Sureban SM, Ali N, Lightfoot SA, Umar S and Houchen CW. DCLK1 facilitates intestinal tumor growth via enhancing pluripotency and epithelial mesenchymal transition. Oncotarget. 2014; 5:9269-9280. doi: 10.18632/ oncotarget.2393.

12. Mirzaei A, Tavoosidana G, Modarressi MH, Rad AA, Fazeli MS, Shirkoohi R, Tavakoli-Yaraki M and Madjd Z. Upregulation of circulating cancer stem cell marker, DCLK1 but not Lgr5, in chemoradiotherapy-treated colorectal cancer patients. Tumour Biol. 2015 ;36:4801-10.

13. Sureban SM, May R, Mondalek FG, Qu D, Ponnurangam S, Pantazis P, Anant S, Ramanujam RP and Houchen CW. Nanoparticle-based delivery of siDCAMKL-1 increases microRNA-144 and inhibits colorectal cancer tumor growth via a Notch-1 dependent mechanism. J Nanobiotechnology. 2011; 9:40.

14. Yan LX, Wu QN, Zhang Y, Li YY, Liao DZ, Hou JH, Fu J, Zeng MS, Yun JP, Wu QL, Zeng YX and Shao JY. Knockdown of miR-21 in human breast cancer cell lines inhibits proliferation, in vitro migration and in vivo tumor growth. Breast Cancer Res. 2011; 13:R2.

15. Tsang JY, Huang YH, Luo MH, Ni YB, Chan SK, Lui PC, $\mathrm{Yu}$ AM, Tan PH and Tse GM. Cancer stem cell markers are associated with adverse biomarker profiles and molecular subtypes of breast cancer. Breast Cancer Res Treat. 2012; 136:407-417.

16. Kwon SY, Bae YK, Gu MJ, Choi JE, Kang SH, Lee SJ, Kim A, Jung HR, Oh HK and Park JY. Neuroendocrine differentiation correlates with hormone receptor expression and decreased survival in patients with invasive breast carcinoma. Histopathology. 2014; 64:647-659.

17. Tan PH, Schnitt SJ, van de Vijver MJ, Ellis IO and Lakhani SR. Papillary and neuroendocrine breast lesions: the WHO stance. Histopathology. 2014; 66:761-770.

18. Lakhani SR, Ellis IO, Schnitt SJ, Tan PH and van de Vijver MJ. (2012). World Health Organisation classification of tumors of the Breast. (Lyon: IARC Press).
19. Wei B, Ding T, Xing Y, Wei W, Tian Z, Tang F, Abraham S, Nayeemuddin K, Hunt K and Wu Y. Invasive neuroendocrine carcinoma of the breast: a distinctive subtype of aggressive mammary carcinoma. Cancer. 2010; 116:4463-4473.

20. Ribeiro AS and Paredes J. P-Cadherin Linking Breast Cancer Stem Cells and Invasion: A Promising Marker to Identify an "Intermediate/Metastable" EMT State. Front Oncol. 2014; 4:371.

21. Park SY, Lee HE, Li H, Shipitsin M, Gelman R and Polyak K. Heterogeneity for stem cell-related markers according to tumor subtype and histologic stage in breast cancer. Clin Cancer Res. 2010; 16:876-887.

22. Tavassoli FA and Devilee P. (2003). World Health Organisation classification of tumors. Pathology and Genetics of tumors of the Breast and Female Genital Organs. (Lyon: IARC Press).

23. Miremadi A, Pinder SE, Lee AH, Bell JA, Paish EC, Wencyk P, Elston CW, Nicholson RI, Blamey RW, Robertson JF and Ellis IO. Neuroendocrine differentiation and prognosis in breast adenocarcinoma. Histopathology. 2002; 40:215-222.

24. Makretsov N, Gilks CB, Coldman AJ, Hayes M and Huntsman D. Tissue microarray analysis of neuroendocrine differentiation and its prognostic significance in breast cancer. Hum Pathol. 2003; 34:1001-1008.

25. van Krimpen C, Elferink A, Broodman CA, Hop WC, Pronk A and Menke M. The prognostic influence of neuroendocrine differentiation in breast cancer: results of a long-term follow-up study. Breast. 2004; 13:329-333.

26. Rovera F, Masciocchi P, Coglitore A, La Rosa S, Dionigi G, Marelli M, Boni L and Dionigi R. Neuroendocrine carcinomas of the breast. Int J Surg. 2008; 6 Suppl 1:S113115.

27. Righi L, Sapino A, Marchio C, Papotti M and Bussolati G. Neuroendocrine differentiation in breast cancer: established facts and unresolved problems. Semin Diagn Pathol. 2010; 27:69-76.

28. Wachter DL, Hartmann A, Beckmann MW, Fasching PA, Hein A, Bayer CM and Agaimy A. Expression of neuroendocrine markers in different molecular subtypes of breast carcinoma. Biomed Res Int. 2014; 2014:408459.

29. Wang J, Wei B, Albarracin CT, Hu J, Abraham SC and $\mathrm{Wu}$ Y. Invasive neuroendocrine carcinoma of the breast: a population-based study from the surveillance, epidemiology and end results (SEER) database. BMC Cancer. 2014; $14: 147$.

30. Zou W, Greenblatt MB, Brady N, Lotinun S, Zhai B, de Rivera H, Singh A, Sun J, Gygi SP, Baron R, Glimcher LH and Jones DC. The microtubule-associated protein DCAMKL1 regulates osteoblast function via repression of Runx2. J Exp Med. 2013; 210:1793-1806.

31. Ferrari N, McDonald L, Morris JS, Cameron ER and Blyth K. RUNX2 in mammary gland development and breast 
cancer. J Cell Physiol. 2013; 228:1137-1142.

32. Owens TW, Rogers RL, Best SA, Ledger A, Mooney AM, Ferguson A, Shore P, Swarbrick A, Ormandy CJ, Simpson PT, Carroll JS, Visvader JE and Naylor MJ. Runx2 is a novel regulator of mammary epithelial cell fate in development and breast cancer. Cancer Res. 2014; 74:52775286. 